\title{
Mechanically controlled reversible photoluminescence response in all-inorganic flexible transparent ferroelectric/mica heterostructures
}

\author{
Ming Zheng (10 ${ }^{1,2}$, Hailing Sun ${ }^{1}$ and K. W. Kwok (1)
}

\begin{abstract}
The ability to reversibly control the luminescent properties of functional materials with diverse external stimuli, such as an electric field, strain, and temperature, is crucial for designing high-performance optical devices. Here, we demonstrate that a purely mechanical strain in a flexible mica substrate triggered by bending can be used to dramatically modify the photoluminescence response of a Pr-doped $\mathrm{Ba}_{0.85} \mathrm{Ca}_{0.15} \mathrm{Ti}_{0.9} \mathrm{Zr}_{0.1} \mathrm{O}_{3}$ epitaxial thin film in a stable and repeatable manner with a large gauge factor of up to 6853. The strong dependence of the photoluminescence performance on the mechanical bending arises from strain-induced variations in the lattice symmetry of the host film and the local crystal field around the $\mathrm{Pr}^{3+}$. In particular, because of the nature of mica, the film structure exhibits excellent antifatigue characteristics after $10^{4}$ bending cycles as well as high optical transparency in the range of $450-780 \mathrm{~nm}$. This study provides a viable route for exploring the correlation between structural symmetry and photoluminescence in ferroelectric thin-film systems and offers new possibilities for developing allinorganic, reconfigurable, transparent and flexible light sources, photodetectors, and wearable sensors.
\end{abstract}

\section{Introduction}

Dramatic advances in the photoluminescence (PL) properties of lanthanide ions embedded in ferroelectric oxides have occurred in recent decades. An example is the repeatable control of PL performance by exerting external physical stimuli (e.g., a magnetic field, an electric field, or temperature difference $)^{1-8}$ to elucidate the fundamental physics of the process and enable use in tunable optical waveguides, display screens, and biological imaging. With the rapid development of emerging technologies, ferroelectric luminescent materials and devices with unique properties, such as being light weight, transparent, flexible and wearable, are highly desirable for practical

\footnotetext{
Correspondence: Ming Zheng (zhengm@mail.ustc.edu.cn) or

K.W. Kwok (apkwkwok@polyu.edu.hk)

'Department of Applied Physics, The Hong Kong Polytechnic University, Kowloon, Hong Kong, China

${ }^{2}$ Department of Physics, Nagoya University, Nagoya 464-8602, Japan
}

applications $^{9-12}$. The conventionally used substrate materials for constructing flexible thin-film devices are polymers, ultrathin glasses, and metal foils ${ }^{12}$. However, the polymers (e.g., polyimide and polyethylene terephthalate) are not thermally stable, thus restricting the growth of high-quality thin films. The glasses are fragile, while the metal foils are nontransparent. The lack of a suitable flexible substrate material has become a bottleneck for this endeavor.

Recently, a breakthrough has been made whereby mechanically exfoliated mica with a thickness below $100 \mu \mathrm{m}$ has been qualified as the substrate material for flexible and transparent oxide heteroepitaxy due to its atomically flat surface, high thermal stability, high transparency, and good mechanical pliability ${ }^{13}$. A number of flexible functional oxide thin films, including ferroelectric $\mathrm{PbZr}_{1-x} \mathrm{Ti}_{x} \mathrm{O}_{3}{ }^{14,15}$ and $\mathrm{BaTi}_{0.95} \mathrm{Co}_{0.05} \mathrm{O}_{3}{ }^{16}$, ferrimagnetic $\mathrm{Fe}_{3} \mathrm{O}_{4}{ }^{17}$, multiferroic $\mathrm{BiFeO}_{3}-\mathrm{CoFe}_{2} \mathrm{O}_{4}{ }^{18}$, metallic 
$\mathrm{MoO}_{2}{ }^{19}$, insulating $\mathrm{VO}_{2}{ }^{20}$, and transparent conducting $\mathrm{Ba}_{0.96} \mathrm{La}_{0.04} \mathrm{SnO}_{3}{ }^{21}$, have been successfully developed on mica substrates. The studies have substantiated the integration of oxide films with flexible mica substrates as an effective strategy for obtaining flexible functional devices. In addition, by bending the mica, the produced mechanical strain can markedly manipulate the physical properties (e.g., Curie temperature, coercive field, and ferromagnetic resonance) of the flexible thin films deposited on $i^{22,23}$. Such a remarkable approach of using mica crystals as substrates makes it possible to prepare flexible and transparent luminescent thin films. If an appropriate rare-earth-doped ferroelectric thin film material is chosen, it is also highly likely to achieve mechanically tunable PL performance for wearable luminescent device applications.

With this as the motivation, we fabricated transparent and flexible Pr-doped $\mathrm{Ba}_{0.85} \mathrm{Ca}_{0.15} \mathrm{Ti}_{0.9} \mathrm{Zr}_{0.1} \mathrm{O}_{3}$ [abbreviated as BCTZ:Pr] epitaxial films on mica substrates and investigated the effect of mechanical strain on their PL properties. The BCTZ host is one of the most widely studied lead-free piezoelectrics in the past decade ${ }^{24} \cdot \operatorname{Pr}^{3+}$ ions are selected as luminescent activators because their $\mathrm{PL}$ emission is very sensitive to the crystallographic symmetry of the BCTZ host through the alteration of the $4 \mathrm{f} 5 \mathrm{~d}$ configuration level ${ }^{25}$. Due to the high sensitivity, the emission of $\operatorname{Pr}^{3+}$ ions has been utilized as a probe for investigating the structural transition of ferroelectric hosts $^{5,7,25}$. Our results reveal that the PL response, including the intensity and splitting of the emission peak, can be adjusted efficiently by mechanical bending with excellent stability and durability. This work provides a promising direction for the design of future all-inorganic transparent wearable optical devices.

\section{Materials and methods}

The $\left(\mathrm{Ba}_{0.85} \mathrm{Ca}_{0.15}\right)_{0.998} \mathrm{Pr}_{0.02} \mathrm{Ti}_{0.9} \mathrm{Zr}_{0.1} \mathrm{O}_{3}$ thin film was grown on a (001)-cut fluorophlogopite mica substrate $\left(\mathrm{KMg}_{3}\left(\mathrm{AlSi}_{3} \mathrm{O}_{10}\right) \mathrm{F}_{2}\right.$, Changchun Taiyuan Co., LTD., China) using the pulsed laser deposition technique. The BCTZ:Pr ceramic target was ablated at $700{ }^{\circ} \mathrm{C}$ under 10 Pa pure oxygen with a $\mathrm{KrF}$ excimer laser $(\lambda=248 \mathrm{~nm})$. After deposition, the film was postannealed in situ at $700{ }^{\circ} \mathrm{C}$ in an oxygen pressure of $10^{3} \mathrm{~Pa}$ for $30 \mathrm{~min}$ to reduce oxygen vacancies and engender good crystalline quality. Then, the sample was cooled to room temperature at a rate of $5^{\circ} \mathrm{C} / \mathrm{min}$.

The crystal structure and epitaxial nature of the asgrown BCTZ:Pr/mica sample were examined by X-ray diffraction (XRD, SmartLab, Rigaku Co., Japan) equipped with $\mathrm{Cu} K_{\alpha 1}(\lambda=1.5406 \AA)$ radiation. The thickness $(120 \mathrm{~nm})$ of the film was determined via X-ray reflectivity. The surface morphology was characterized using scanning probe microscopy (SPM, Asylum MFP 3D Infinity). The transmittance spectra were recorded using a UV-vis spectrometer (Shimadzu Co.). The PL excitation and emission spectra were measured using an Edinburgh FLSP920 spectrophotometer with a 450-nm xenon arc lamp (Xe900) as the excitation source. Prior to the measurements, the two-dimensional layered mica substrate was mechanically exfoliated, like graphene, to a thickness of $10 \mu \mathrm{m}$ using a sharp blade to acquire good mechanical flexibility. By taping the BCTZ:Pr/mica sample onto the polyester convex molds with different radii of curvature (ROCs), a tunable tensile mechanical strain was induced in the BCTZ:Pr thin film. Note that the sample was firmly attached to the mold to avoid slipping and fracturing during the measurement. All measurements were performed at ambient temperature.

\section{Results and discussions}

Figure 1a shows a typical out-of-plane XRD $\theta-2 \theta$ scan spectrum of the BCTZ:Pr/mica structure. The appearance of only BCTZ:Pr $(l l l)(l=1,2)$ reflections indicates the preferred orientation growth of the BCTZ:Pr film with a single-phase structure. The out-of-plane interplanar spacing $d_{111}(2.323 \AA)$ of the BCTZ:Pr film is larger than that of the BCTZ:Pr bulk value ( $2.321 \AA)$, suggesting that the film has an out-of-plane tensile strain (0.086\%) accompanied by an in-plane compressive strain. XRD $\phi$ scans were performed on the BCTZ:Pr (101) and mica (202) reflection peaks to further reveal the in-plane crystallographic orientation of the film with the substrate. As shown in Fig. 1b, compared with the trifold symmetry of mica, sixfold symmetric reflection peaks are observed at $60^{\circ}$ intervals from the BCTZ:Pr due to the occurrence of two sets of structural domains in the films. The epitaxial relationship of the film with respect to the substrate is determined to be $(111)_{\mathrm{BCTZ}: \mathrm{Pr}}||(001)_{\mathrm{Mica}}$ and $[01-1]_{\mathrm{BCTZ}}$ Pr ||$[010]_{\text {Mica. }}$ The BCTZ:Pr film also exhibits a flat surface, sharp interface, and uniform thickness, as evidenced by the surface morphology image (inset) and the clear interference pattern of the X-ray reflectivity curve (Fig. 1c). A full width at half maximum of $\sim 0.51^{\circ}$ is observed, indicating excellent crystallinity in the BCTZ:Pr film. Moreover, the transmittance of the BCTZ:Pr/mica structure is over $70 \%$ in the visible light region ranging from 450 to $780 \mathrm{~nm}$ (Fig. 1d), which is higher than that of other ferroelectric/mica heterostructures ${ }^{14,16}$. As shown in the insets of Fig. 1d, the sample possesses excellent transparency and mechanical flexibility. All the above characterizations demonstrate a high-quality surface and good epitaxial heterointerface of the flexible and transparent BCTZ:Pr/mica structure, which provide strong elastic coupling at the interface of the structure either in a flat or bent state.

To explore the mechanical strain effect on the PL response, predesigned polyester convex molds with various ROCs were employed to introduce different bending 

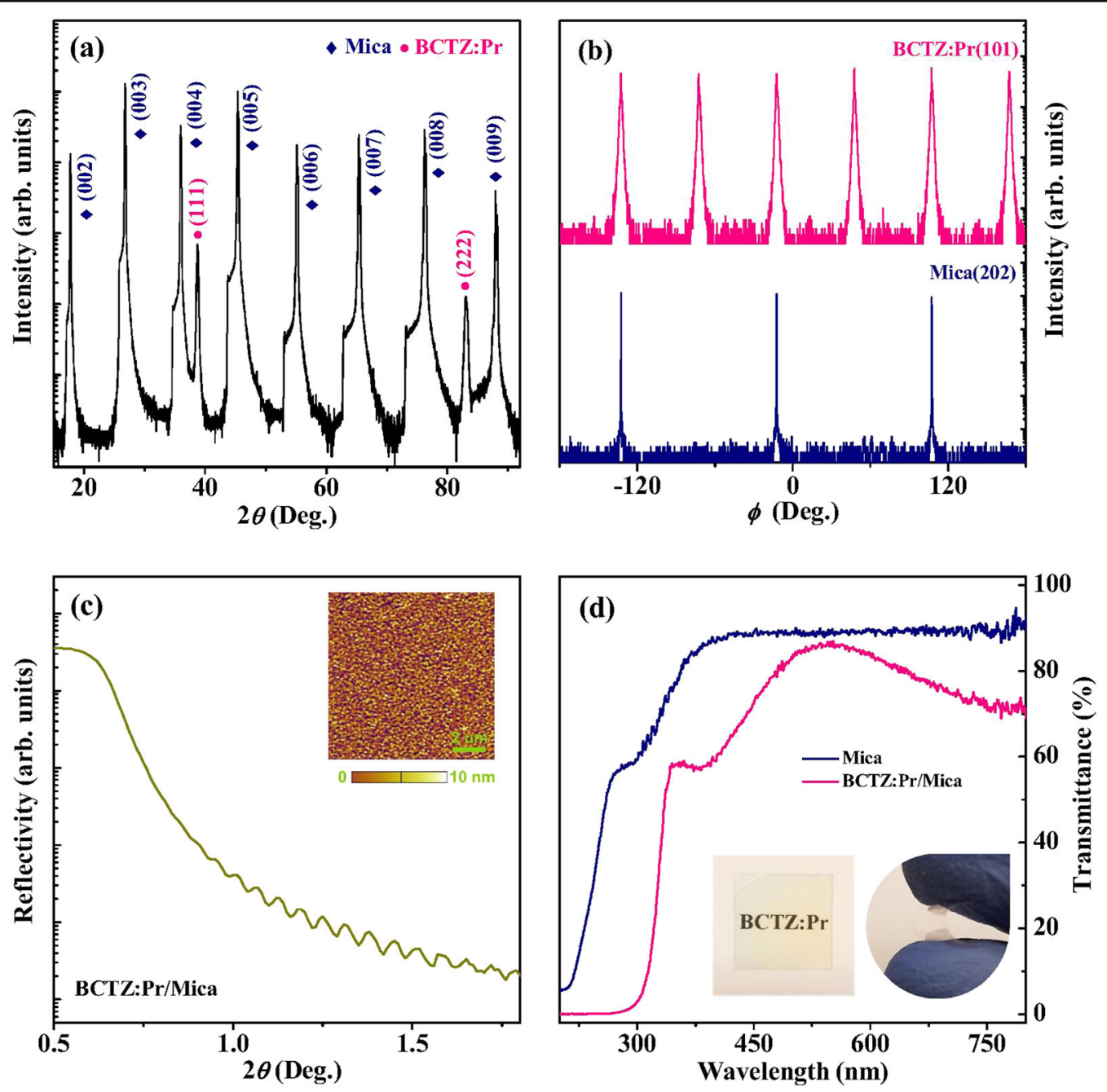

Fig. 1 a XRD $\theta-2 \theta$ scan spectrum of the BCTZ:Pr/mica structure. $\mathbf{b}$ XRD $\phi$ scans of the BCTZ:Pr (101) and mica (202) reflection peaks. c X-ray reflectivity curve of the BCTZ:Pr/mica structure. The inset shows the surface morphology of the BCTZ:Pr film. $\mathbf{d}$ Optical spectra of the mica substrate and the BCTZ:Pr/mica structure. The insets show optical images of the flat and bent BCTZ:Pr/mica samples

strains in the film by attaching the flexible BCTZ:Pr/mica structure onto them (inset of Fig. 2a). The photoluminescence excitation spectrum of the flat BCTZ:Pr/ mica structure monitored at $578 \mathrm{~nm}$ was first measured, giving the results shown in Fig. 2a. Three excitation peaks corresponding to the ${ }^{3} P_{0} \rightarrow{ }^{3} H_{5},{ }^{3} P_{1} \rightarrow{ }^{3} H_{6}$ and ${ }^{3} P_{0} \rightarrow$ ${ }^{3} F_{2}$ transitions of $\mathrm{Pr}^{3+}$ are observed at 440, 468, and $495 \mathrm{~nm}$, respectively. Based on the results, the PL emission spectra of the structure in different bent states (with different ROCs) as well as the flat state were recorded under an excitation of $468 \mathrm{~nm}$. As shown in Fig. 2b, similar to earlier reports on other Pr-doped materials ${ }^{24,26}$, the BCTZ:Pr film exhibits strong PL emissions of $\operatorname{Pr}^{3+}$ ions. The emission peaks centered at $549,578,596,616$, and $647 \mathrm{~nm}$ originate from the ${ }^{3} P_{0} \rightarrow{ }^{3} H_{5},{ }^{3} P_{1} \rightarrow{ }^{3} H_{6}$, ${ }^{1} D_{2} \rightarrow{ }^{3} H_{4}, \quad{ }^{3} P_{0} \rightarrow{ }^{3} H_{6}$, and ${ }^{3} P_{0} \rightarrow{ }^{3} F_{2}$ transitions, respectively. The multiplets associated with these transitions stem from crystal field-induced Stark splitting, which has been observed for $\mathrm{Pr}^{3+}$ ions in $\mathrm{Ba}_{0.77} \mathrm{Ca}_{0.23} \mathrm{TiO}_{3}$ and $\mathrm{CaTiO}_{3}$ hosts ${ }^{25,27}$. Obviously, the PL response is strongly dependent on the bending radius (i.e., radius of curvature (ROC)) for all of the emission peaks from green to red. It is noted that the strongest emission peak corresponding to the ${ }^{3} P_{1} \rightarrow{ }^{3} H_{6}$ transition split into two peaks upon bending. At a ROC $>5 \mathrm{~mm}$, the split peak at a low wavelength is stronger than the other one at a high wavelength, whereas it becomes weaker at a ROC $<5 \mathrm{~mm}$. The bending-induced relative change in the PL intensity $(\Delta I / I)$ of the strongest emission peak as a function of the ROC is shown in Fig. 3a. Here, $\Delta I / I$ is defined as $\Delta I / I=$ $[I(\mathrm{ROC})-I(\mathrm{ROC}=\infty)] / I(\mathrm{ROC}=\infty)$. The $\Delta I / I$ first decreases gradually with decreasing $\mathrm{ROC}$ and then increases sharply after reaching a minimum value at a ROC $=5 \mathrm{~mm}$. As illustrated in the inset of Fig. 3b, a 

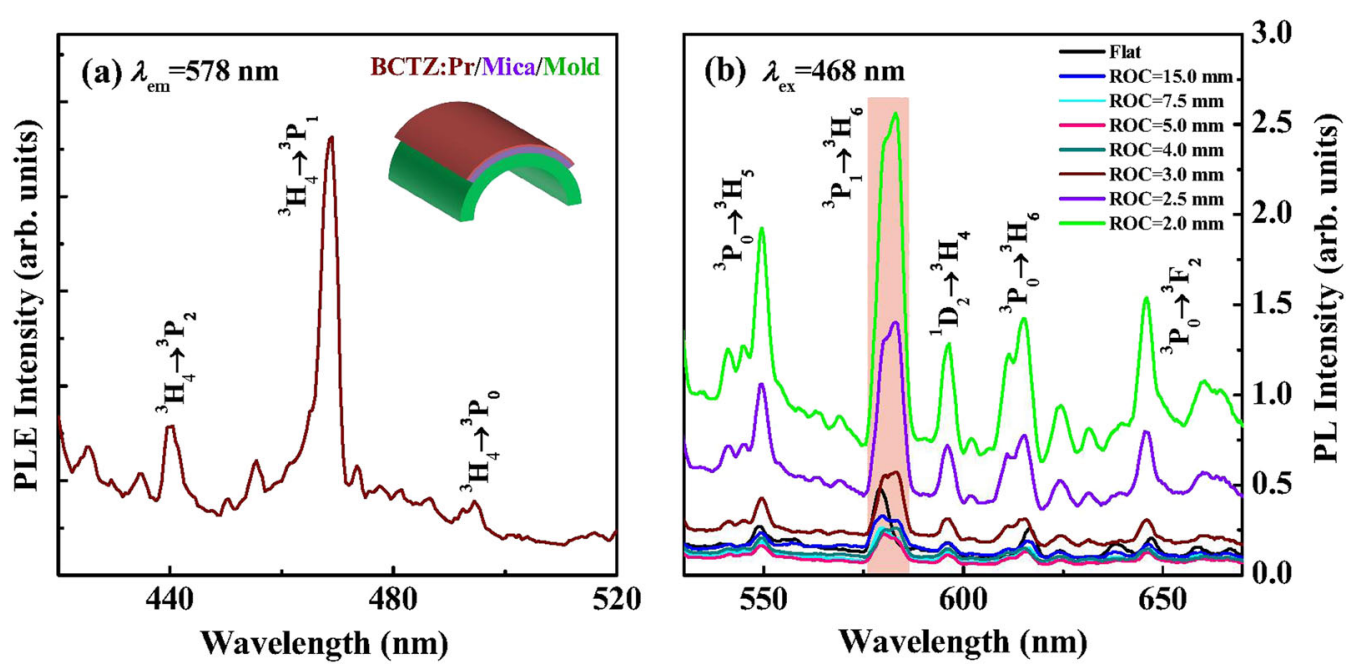

Fig. 2 The PL a excitation spectrum and $\mathbf{b}$ emission spectra of the BCTZ:Pr/mica structure at various ROCs. The inset in a shows the schematic of the bending configuration of the structure on a convex mold
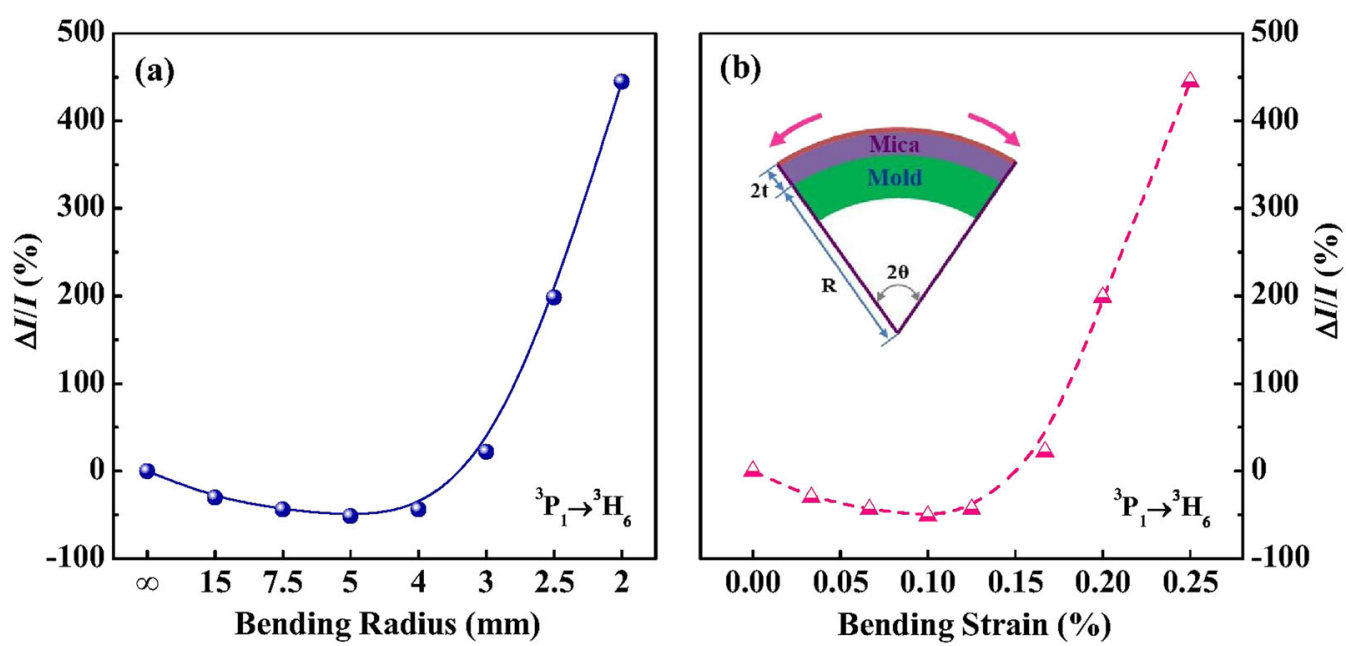

Fig. 3 The relative change in PL intensity $(\Delta / / I)$ as a function of $\mathbf{a}$ the bending radius and $\mathbf{b}$ the bending strain for the structure. The inset in $\mathbf{b}$ shows the illustration of the structure with a tensile in-plane strain under outward bending

tensile in-plane mechanical strain is introduced in the BCTZ:Pr film upon bending the mica substrate outward. It is thus inferred that a close relationship between the PL performance and bending-induced mechanical strain exists.

As reported by Zhao et al. and Zhang et al. ${ }^{28,29}$, the bending strain $\varepsilon$ in the BCTZ:Pr film can be described as $\varepsilon$ $=[(t+R) \theta-R \theta] / R \theta=t / R$, where $t$ is the half of the total thickness of the film and the mica substrate and $R$ is the ROC (inset of Fig. $3 \mathrm{~b}$ ). The bending strain at each ROC was then calculated, and its relationship with $\Delta I / I$ is presented in Fig. 3b. Similar to the dependence on the ROC, the $\Delta I / I$ decreases first with increasing $\varepsilon$ and then increases abruptly after reaching a minimum value of
$-52 \%$ at $\varepsilon=0.1 \%$. It is worth noting that the value of $\Delta I / I$ reaches a very large value of $445 \%$ at $\varepsilon=0.25 \%$. It is known that the PL response is closely related to the structural symmetry of the host and the crystal field around rare-earth ions ${ }^{30,31}$. Based on the Judd-Ofelt (J-O) theory, a decrease in the structural symmetry of the $\mathrm{BCTZ}$ host triggers an increase in uneven components of the crystal field around the $\operatorname{Pr}^{3+}$ dopant ions. The uneven crystal field can mix opposite-parity states into the $4 \mathrm{f}$ configuration level and enhance the electronic coupling between $4 \mathrm{f}$ energy levels and the higher $4 \mathrm{f} 5 \mathrm{~d}$ configuration, thereby leading to an increase in the $4 \mathrm{f}-4 \mathrm{f}$ electric dipolar transition probabilities of $\mathrm{Pr}^{3+}$ ions ${ }^{32}$. In other words, the lower the structural symmetry, the stronger the 

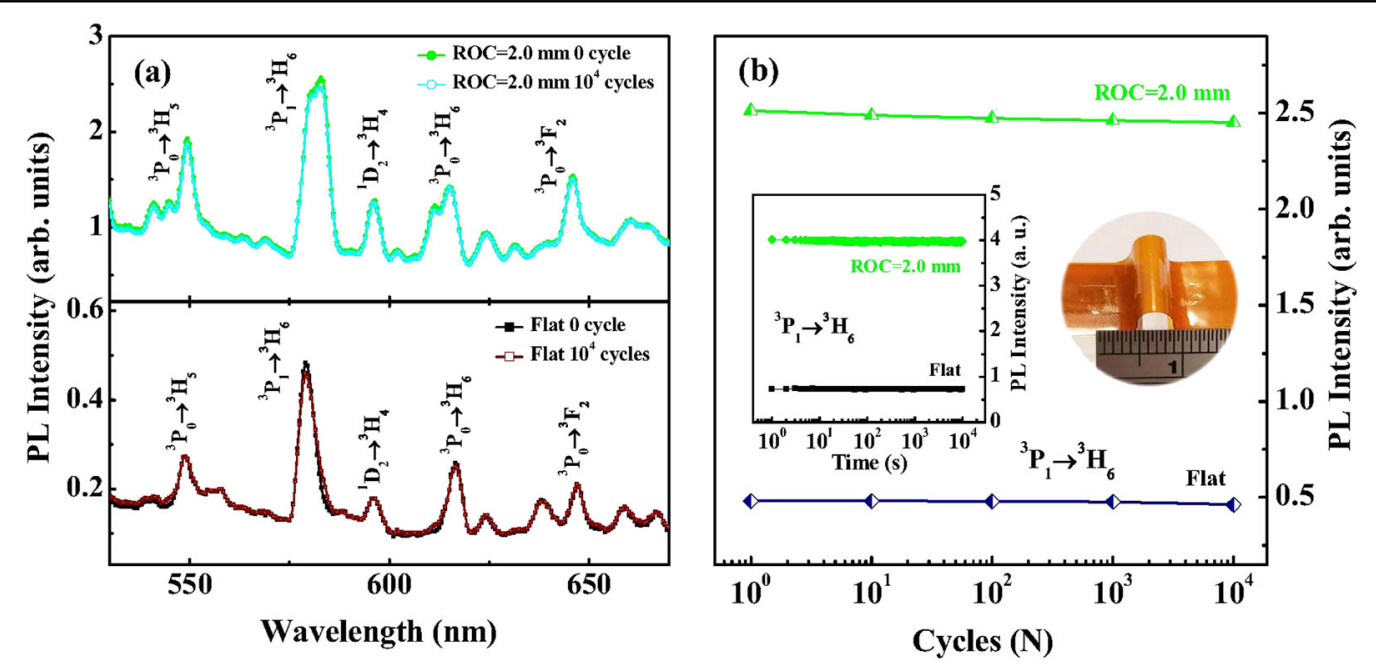

Fig. 4 a The PL emission spectra of the BCTZ:Pr/mica structure, both in the flat $(R O C=\infty)$ and bent $(R O C=2 \mathrm{~mm})$ states, before and after $10^{4} \mathrm{cycles}$ of bending. $\mathbf{b}$ The PL intensity plotted as a function of the bending cycle. The insets show the retention measurement of the PL intensity at the flat and bent states (left) and a schematic of the cyclic bending process (right)

PL emission. This result has been verified for many lanthanide-doped ferroelectric ceramic bulk and thin film materials, where the PL response is mediated by the variation in structural symmetry of the host through the electric-field-induced biaxial strain, polarization, or phase transition $^{3,5-8}$. As discussed above (Fig. 1a), an initial compressive in-plane strain (i.e., epitaxial strain) is produced in the BCTZ:Pr film because of a lattice mismatch. Using the Poisson relation $\varepsilon_{z z}=-2 v /(1-v) \varepsilon_{x x}{ }^{33-35}$ and Poisson ratio $v=0.3^{36}$, the epitaxial strain is estimated to be $-0.1 \%$. This compressive epitaxial strain can be canceled out by bending the substrate outward (inset of Fig. 3b). As shown in Fig. 3a, b, the effective in-plane strain $\varepsilon_{\text {eff }}$ (i.e., the sum of the epitaxial strain and bending strain) of the film should become almost zero at a $\mathrm{ROC}=5 \mathrm{~mm}$, thus leading to the smallest distortion, the highest structural symmetry, and then the weakest PL emission. For a ROC $>5 \mathrm{~mm}$ (in-plane compressive) or ROC $<5 \mathrm{~mm}$ (inplane tensile), the PL intensity is increased due to a reduction in the structural symmetry of the host. The mechanically tunable PL response is attributed to strainmediated symmetries of the host and crystal fields around $\mathrm{Pr}^{3+}$, which vary the radiative transition probabilities of the lanthanide ions. An ultralarge gauge factor or figure of merit (for describing the effectiveness of the strain tunability of PL intensity) is $(\Delta I / I) / \Delta \varepsilon=\{[I(\mathrm{ROC}=2 \mathrm{~mm})-$ $I(\mathrm{ROC}=5 \mathrm{~mm})] / I(\mathrm{ROC}=5 \mathrm{~mm})\} /[\varepsilon(\mathrm{ROC}=2 \mathrm{~mm})-\varepsilon$ $(\mathrm{ROC}=5 \mathrm{~mm})]=1028 \% / 0.15 \%=6853$. Such a remarkable PL tuning effect by mechanical strain has not been reported thus far; therefore, the flexible BCTZ:Pr thin films on mica substrates should be a promising material for novel highly sensitive flexible luminescent device applications.
From the perspective of practical applications, a long durability and high stability are essential for a long operational lifetime. In this work, the durability was assessed by investigating the PL response of the flexible BCTZ:Pr/mica structure subjected to cyclic bending (up to $10^{4}$ cycles), while the stability was evaluated by monitoring the PL intensity of the strongest emission peak, both in the flat $(\mathrm{ROC}=\infty)$ and bent $(\mathrm{ROC}=2 \mathrm{~mm})$ states, as a function of time. A photograph illustrating the cyclic bending is shown in the inset of Fig. 4b. As shown in Fig. 4a, the PL emission spectra of the film, both in the flat and bent states, remain almost unaltered after $10^{4}$ cycles of bending. Similarly, the PL intensities of the strongest emission peak in the flat and bent states decrease only slightly during the cyclic bending and retain more than $95 \%$ of the initial values after $10^{4}$ cycles of bending (Fig. 4b). These clearly demonstrate the switching endurance and excellent durability of the BCTZ:Pr/ mica structure. It has been reported that heteroepitaxy involving two-dimensional materials (e.g., mica) can be categorized as van der Waals epitaxy ${ }^{12,13,18-20}$. The excellent durability of the PL response of the BCTZ:Pr films upon bending the mica can be attributed to the weak van der Waals interaction between the film and twodimensional layered substrate instead of the strong chemical bonding, which can reduce the substrate clamping effect $^{12,13}$. The PL retention properties of the structure in both states are depicted in the inset of Fig. 4b. No obvious decay of the PL intensities is observed over a long period of $10^{4} \mathrm{~s}$. Compared with the electric/magnetic field-driven luminescent devices, the mechanical strain-induced dynamic and continuous PL switching in the flexible BCTZ:Pr/mica structure indicates the possibility of 


\section{Bending}
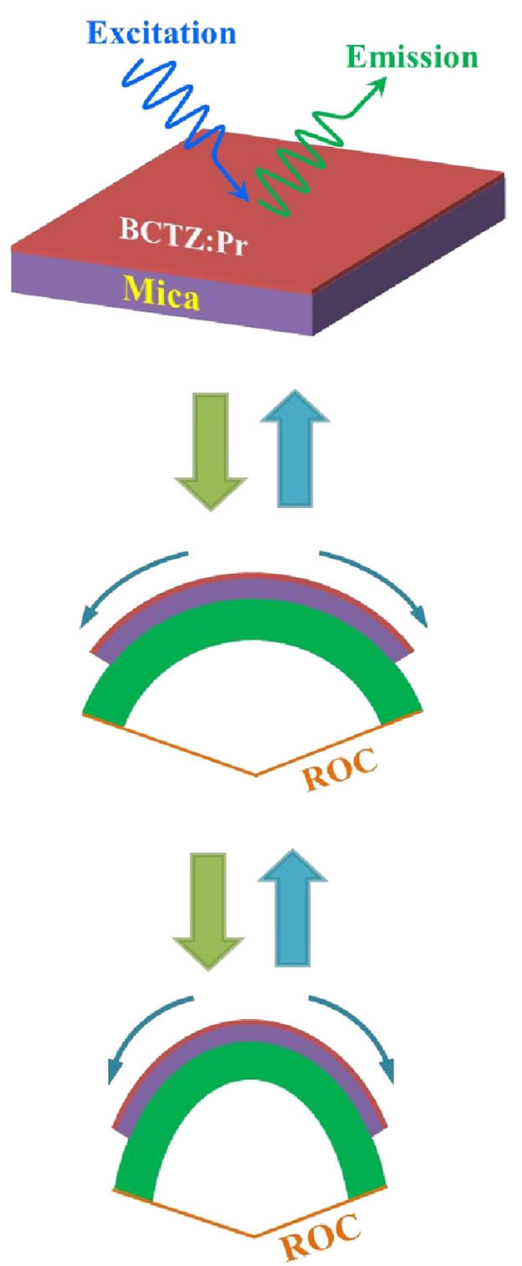

\section{PL Signals}
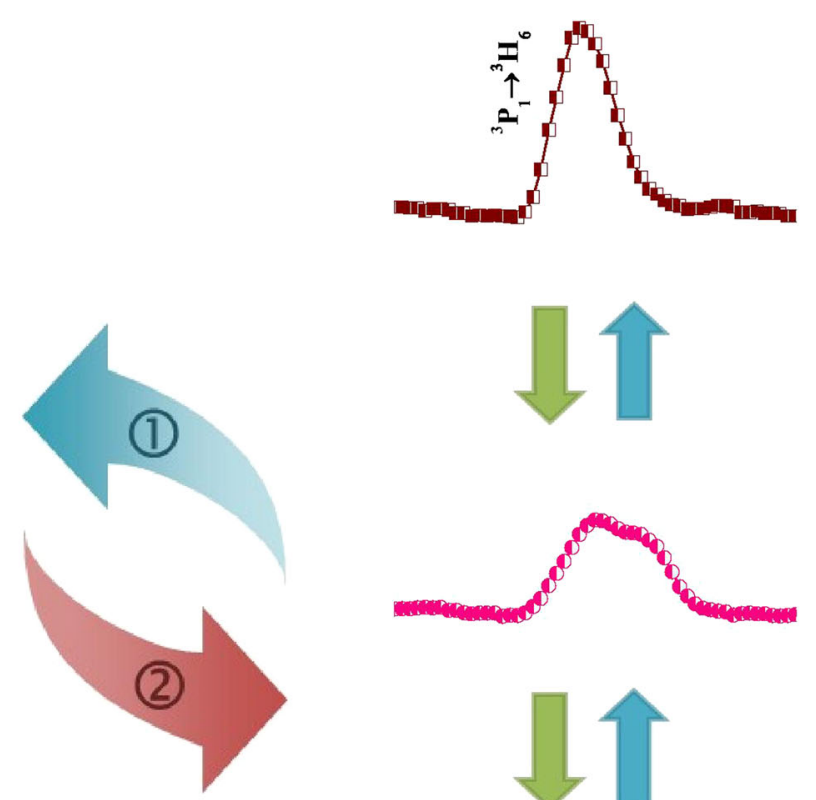

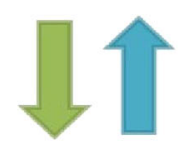

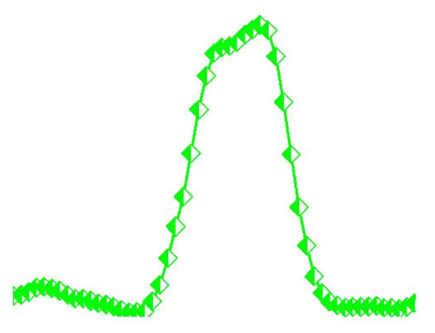

Fig. 5 Illustrations of the transparent wearable mechanic-luminescent sensing system for the flexible BCTZ:Pr/mica structure. In process (1), the PL signal change can be used to detect the curvature variation. In process (2), changing the curvature can obtain a specific PL signal

practical use for high-performance wearable optical devices due to its excellent stability, reliability, and antifatigue behavior.

The tunable mechanical strain effect due to bending provides great potential for the design and development of transparent wearable mechanic-luminescent sensing systems. As illustrated in Fig. 5, we can detect and adjust the ROC of the device by recording the evolution of the PL spectra (e.g., the intensity and wavelength of the emission peak), corresponding to process (1), which can be adopted in flexible optical deformation sensors. On the other hand, a specific PL signal can be obtained through accurately controlling the ROC (i.e., process (2), which provides an intriguing approach for flexible display lighting, optical filters, and medical imaging. Although we have proven the validity of the mechanically controlled PL response in BCTZ:Pr films by using flexible mica as a substrate, we anticipate that similar modulation is achievable in other perovskite oxide thin films, such as $\mathrm{BaTiO}_{3}{ }^{3},(\mathrm{Bi}, \mathrm{Na}) \mathrm{TiO}_{3}{ }^{4,6}$, and $(\mathrm{Li}, \mathrm{Na}, \mathrm{K}) \mathrm{NbO}_{3}{ }^{32}$, doped with lanthanide ions and epitaxially grown on the mica substrates. In addition, the mechanical strain in tension/ compression induced by outward/inward bending can also be diverted into $\mathrm{NaGdF}_{4}$ and metal halide nanocrystals and nanowires by implementing them on mica substrates $^{2,37-39}$, and these structures are also promising candidates for mechanically tunable PL properties.

Table 1 summarizes the relative change of PL emission intensity of several lanthanide ions when the corresponding ferroelectric hosts are under different external 
Table 1 PL response under external stimuli for several lanthanide-doped ferroelectric elements studied extensively

\begin{tabular}{|c|c|c|c|c|c|c|c|}
\hline Reference & This work & Zheng et al. ${ }^{7}$ & Wang et al. ${ }^{8}$ & Hao et al. ${ }^{3}$ & Sun et al. ${ }^{5}$ & Zhang et al. ${ }^{25}$ & Khatua et al. ${ }^{4}$ \\
\hline \multirow[t]{2}{*}{ Host material } & $\mathrm{BCTZ}$ & $\mathrm{BCTZ}$ & $\mathrm{BaTiO}_{3}$ & $\mathrm{BaTiO}_{3}$ & $\mathrm{BCTZ}$ & $\mathrm{Ba}_{0.77} \mathrm{Ca}_{0.23} \mathrm{TiO}_{3}$ & $\mathrm{Na}_{0.5} \mathrm{Bi}_{0.5} \mathrm{TiO}_{3}-\mathrm{BaTiO}_{3}$ \\
\hline & Thin film & Thin film & Thin film & Thin film & Bulk ceramic & Bulk ceramic & Bulk ceramic \\
\hline Lanthanide ions & $\operatorname{Pr}$ & $\operatorname{Pr}$ & $\mathrm{Yb} / \mathrm{Er}$ & $\mathrm{Yb} / \mathrm{Er}$ & $\operatorname{Pr}$ & $\operatorname{Pr}$ & Eu \\
\hline Substrate & Mica & PMN-PT & PMN-PT & $\mathrm{SrTiO}_{3}$ & - & - & - \\
\hline External stimulus & Mechanical strain & Electric field & Electric field & Electric field & Electric field & Electric field & Electric field \\
\hline$\Delta / / I(\%)$ & 1028 & 6.6 & 15 & 170 & 22 & 30 & 12.8 \\
\hline Emission peak (nm) & 578 & 611 & 525 & 523 & 650 & 611 & 593 \\
\hline
\end{tabular}

stimuli. It is clear from the table that the BCTZ:Pr/mica system has the best performance metrics among all the lanthanide-doped ferroelectric elements. It is interesting to compare the BCTZ:Pr/mica structure with the BCTZ: $\mathrm{Pr} / \mathrm{Pb}\left(\mathrm{Mg}_{1 / 3} \mathrm{Nb}_{2 / 3}\right) \mathrm{O}_{3}-\mathrm{PbTiO}_{3}(\mathrm{PMN}-\mathrm{PT})$ structure in our previous study ${ }^{7}$. Both systems allow a gradual and continuous evolution in the strain of the films, which enables us to precisely fine-tune the PL response. In the case of the BCTZ:Pr/PMN-PT structure, the PL modulation $(6.6 \%)$ is mediated by the external electric-field-generated relatively small piezostrain $(0.055 \%)$ of the PMN-PT, where we only shed light on lattice-coupled PL performance for the lateral compressively strained thin films (considering the sum of the epitaxial strain and piezostrain) due to much smaller lattice parameters of the $\mathrm{La}_{0.7} \mathrm{Sr}_{0.3} \mathrm{MnO}_{3}$ buffer layer than those of the overlying BCTZ:Pr film. In the BCTZ:Pr/mica structure, the strain switching for PL is produced by purely mechanical bending without being driven by an electric signal. The inplane strain state of the thin film can be changed from compressive to tensile (considering the sum of the epitaxial strain and bending strain) due to the good pliability of the mica, which gives rise to a rich physical process of energy transition and a large PL tuning effect. We note that the gauge factor (6853) of the BCTZ:Pr film on mica is much larger than that (120) on PMN-PT. This finding is ascribed to a reduction in the substrate clamping effect induced by the weak van der Waals interaction between the film and two-dimensional layered mica substrate ${ }^{12,13}$.

\section{Conclusions}

In summary, we report a purely mechanical straininduced PL and repeatable control of the PL response using a simple BCTZ:Pr/mica epitaxial structure. Owing to the nature of mica, the structure possesses high optical transmittance and excellent mechanical flexibility. The PL switching shows no noticeable decay after being bent to a ROC of $2 \mathrm{~mm}$ for $10^{4}$ cycles, demonstrating the robust functionality of the BCTZ:Pr films against mechanical bending. Our finding is informative for achieving mechanically controlled PL properties in lattice-sensitive ferroelectric oxides and designing next-generation allinorganic, reconfigurable, light weight, transparent, and flexible passive luminescent devices. The high-quality heteroepitaxy on mica is expected to open a new and simple approach for expanding the material database, such as colossal magnetoresistive manganites and topological insulators, for future soft technology.

\section{Acknowledgements}

This work was supported by the Research Grants Council of the Hong Kong Special Administrative Region (PolyU 152753/16E) and The Hong Kong Polytechnic University (1-ZVGH).

Conflict of interest

The authors declare that they have no conflict of interest.

Publisher's note

Springer Nature remains neutral with regard to jurisdictional claims in published maps and institutional affiliations.

Received: 5 May 2019 Revised: 10 June 2019 Accepted: 14 June 2019. Published online: 27 September 2019

\section{References}

1. Zhang, Y., Jie, W., Chen, P., Liu, W. \& Hao, J. Ferroelectric and piezoelectric effects on the optical process in advanced materials and devices. Adv. Mater. 30, 1707007 (2018)

2. Liu, Y. X., Wang, D. S., Shi, J. X., Peng, Q. \& Li, Y. D. Magnetic tuning of upconversion luminescence in lanthanide-doped bifunctional nanocrystals. Angew. Chem., Int. Ed. 52, 4366 (2013).

3. Hao, J. H., Zhang, Y. \& Wei, X. H. Electric-induced enhancement and modulation of upconversion photoluminescence in epitaxial $\mathrm{BaTO}_{3}: \mathrm{Yb} / \mathrm{Er}$ thin films. Angew. Chem., Int. Ed. 50, 6876 (2011).

4. Khatua, D. K., Kalaskar, A. \& Ranjan, R. Tuning photoluminescence response by electric field in electrically soft ferroelectrics. Phys. Rev. Lett. 116, 117601 (2016)

5. Sun, H. L., Wu, X., Chung, T. H. \& Kwok, K. W. In-situ electric field-induced modulation of photoluminescence in $\mathrm{Pr}$-doped $\mathrm{Ba}_{0.85} \mathrm{Ca}_{0.15} \mathrm{~T}_{0.90} \mathrm{Zr}_{0.10} \mathrm{O}_{3}$ leadfree ceramics. Sci. Rep. 6, 28677 (2016).

6. Sun, H. L., Wu, X., Peng, D. F. \& Kwok, K. W. Room-temperature large and reversible modulation of photoluminescence by in situ electric field in ergodic relaxor ferroelectrics. ACS Appl. Mater. Interfaces 9, 34042 (2017).

7. Zheng, M., Sun, H. L., Chan, M. K. \& Kwok, K. W. Reversible and nonvolatile tuning of photoluminescence response by electric field for reconfigurable luminescent memory devices. Nano Energy 55, 22 (2019). 
8. Wang, F. F. et al. In situ reversible tuning of photoluminescence of an epitaxial thin film via piezoelectric strain induced by a $\mathrm{Pb}\left(\mathrm{Mg}_{1 / 3} \mathrm{Nb}_{2 / 3}\right) \mathrm{O}_{3}-\mathrm{PbTiO}_{3}$ single crystal. J. Mater. Chem. C 5, 9115 (2017).

9. Mönch, l. et al. Rolled-up magnetic sensor: nanomembrane architecture for inflow detection of magnetic objects. ACS Nano 5, 7436 (2011).

10. Zhang, L., Fairbanks, M. \& Andrew, T. L. Rugged textile electrodes for wearable devices obtained by vapor coating off-the-shelf, plain-woven fabrics. Adv. Funct. Mater. 27, 1700415 (2017).

11. Yang, T., Xie, D., Li, Z. \& Zhu, H. Recent advances in wearable tactile sensors: materials, sensing mechanisms, and device performance. Mater. Sci. Eng., Rep. 115, 1 (2017)

12. Bitla, Y. \& Chu, Y. H. MICAtronics: a new platform for flexible X-tronics. FlatChem 3, 26 (2017).

13. Chu, Y. H. Van der Waals oxide heteroepitaxy. NPJ Quantum Mater. 2, 67 (2017).

14. Wang, D., Yuan, G. L., Hao, G. \& Wang, Y. All-inorganic flexible piezoelectric energy harvester enabled by two-dimensional mica. Nano Energy 43, 351 (2018).

15. $\mathrm{Li}, \mathrm{M}$. et al. Ferroelectricity of flexible $\mathrm{Pb}\left(\mathrm{Zr}_{0.53} \mathrm{Ti}_{0.47}\right) \mathrm{O}_{3}$ thin film at high temperature. Acta Phys. Sin. 68, 087302 (2019).

16. Yang, Y. et al. Flexible, semitransparent, and inorganic resistive memory based on $\mathrm{BaTi}_{0.95} \mathrm{CO}_{0.05} \mathrm{O}_{3}$ film. Adv. Mater. 29, 1700425 (2017).

17. Zheng, W. C., Zheng, D. X., Wang, Y. C., Jin, C. \& Bai, H. L. Uniaxial strain tuning of the Verwey transition in flexible $\mathrm{Fe}_{3} \mathrm{O}_{4} /$ muscovite epitaxial heterostructures. Appl. Phys. Lett. 113, 142403 (2018).

18. Amrillah, T. et al. Flexible multiferroic bulk heterojunction with giant magnetoelectric coupling via van der Waals epitaxy. ACS Nano 11, 6122 (2017).

19. $\mathrm{Ma}, \mathrm{C}$. et al. Van der Waals epitaxy of functional $\mathrm{MoO}_{2}$ film on mica for flexible electronics. Appl. Phys. Lett. 118, 253104 (2016).

20. Li, C. et al. van der Waal epitaxy of flexible and transparent $\mathrm{VO}_{2}$ film on muscovite. Chem. Mater. 28, 3914 (2016).

21. Sun, W. et al. High optical transmittance and anomalous electronic transport in flexible transparent conducting oxides $\mathrm{Ba}_{0.96} \mathrm{La}_{0.04} \mathrm{SnO}_{3}$ thin films. Ceram. Int. 44, 18001 (2018).

22. Liu, J. et al. Mechanically tunable magnetic properties of flexible $\mathrm{SrRuO}_{3}$ epitaxial thin films on mica substrates. Adv. Electron. Mater. 4, 1700522 (2018).

23. Liu, W. et al. Mechanical strain-tunable microwave magnetism in flexible $\mathrm{CuFe}_{2} \mathrm{O}_{4}$ epitaxial thin film for wearable sensors. Adv. Funct. Mater. 28, 1705928 (2018).
24. Liu, W. \& Ren, X. B. Large piezoelectric effect in Pb-free ceramics. Phys. Rev. Lett. 103, 257602 (2009).

25. Zhang, $\mathrm{P}$. et al. $\mathrm{Pr}^{3+}$ photoluminescence in ferroelectric $\left(\mathrm{Ba}_{0.77} \mathrm{Ca}_{0.23}\right) \mathrm{TiO}_{3}$ ceramics: sensitive to polarization and phase transitions. Appl. Phys. Lett. 92, 222908 (2008).

26. Huang, Y. et al. The down-conversion and up-conversion photoluminescence properties of $\mathrm{Na}_{0.5} \mathrm{Bi}_{0.5} \mathrm{TiO}_{3}: \mathrm{Yb}^{3+} / \mathrm{Pr}^{3+}$ ceramics. J. Appl. Phys. 118, 044101 (2015).

27. Boutinaud, P., Pinel, E., Dubois, M., Vink, A. P. \& Mahiou, R. UV-to-red relaxation pathways in $\mathrm{CaTiO}_{3}: \mathrm{Pr}^{3+}$. J. Lumin. 111, 69 (2005).

28. Zhao, J. et al. Ultra-sensitive strain sensors based on piezoresistive nanographene films. Appl. Phys. Lett. 101, 063112 (2012).

29. Zhang, Y. et al. Flexible quasi-two-dimensional $\mathrm{CoFe}_{2} \mathrm{O}_{4}$ epitaxial thin films for continuous strain tuning of magnetic properties. ACS Nano 10, 1481 (2017).

30. Judd, B. R. Optical absorption intensities of rare-earth ions. Phys. Rev. 127, 750 (1962).

31. Ofelt, G. S. Intensities of crystal spectra of rare-earth ions. J. Chem. Phys. 37, 511 (1962).

32. Wu, X., Lau, C. M. \& Kwok, K. W. Photoluminescence properties of Er/Pr-doped $\mathrm{K}_{0.5} \mathrm{Na}_{0.5} \mathrm{NbO}_{3}$ ferroelectric ceramics. J. Am. Ceram. Soc. 98, 2139 (2015).

33. Zheng, M. et al. Ferroelastic strain control of multiple nonvolatile resistance tuning in $\mathrm{SrRuO}_{3} / \mathrm{PMN}-\mathrm{PT}(111)$ multiferroic heterostructures. Appl. Phys. Lett. 110, 182403 (2017).

34. Zheng, M. et al. Optically and electrically co-controlled resistance switching in complex oxide heterostructures. Appl. Phys. Lett. 111, 172901 (2017).

35. Zheng, M. et al. Ferroelastically and magnetically co-coupled resistive switching in $\mathrm{Nd}_{0.5} \mathrm{Sr}_{0.5} \mathrm{MnO}_{3} / \mathrm{PMN}-\mathrm{PT}(011)$ multiferroic heterostructures. Appl. Phys. Lett. 112, 123502 (2018).

36. Li, Y. W. \& Li, F. X. In situ observation of electric field induced crack propagation in $\mathrm{BaTiO}_{3}$ crystals along the field direction. Scr. Mater. 67, 601 (2012).

37. Lyu, L. et al. Near-infrared light-mediated rare-earth nanocrystals: recent advances in improving photon conversion and alleviating the thermal effect. NPG Asia Mater. 10, 685 (2018).

38. Hasegawa, Y., Kitagawa, Y. \& Nakanishi, T. Effective photosensitized, electrosensitized, and mechanosensitized luminescence of lanthanide complexes. NPG Asia Mater. 10, 52 (2018).

39. Utama, M. et al. Incommensurate van der Waals epitaxy of nanowire arrays: a case study with ZnO on muscovite mica substrates. Nano Lett. 12, 2146 (2012). 